\title{
Virtual Reality Support for Dental Treatment Premedication
}

\author{
Tomasz ('Tomi') Kupka
}

DMD, PhD, Dental surgery, Ul. Kochłowicka 3, 41-706 Ruda Śląska, Poland

Corresponding author email: kupkatomasz1@gmail.com

Received: 09 December 2020 / Revised: 04 January 2021 / Accepted: 14 January 2021 / Published: 19 January 2021

\begin{abstract}
The objective is to short review the current state of the art of virtual reality medical application in term of minimal invasive dentistry. An outline of the most important aspects of virtual reality has been created, and a subsequent literature search for articles related was conducted. The current state of the art of virtual reality includes a variety of applications, among others premedication, analgesics, anaesthetics plays an important role in informatics medicine. There is a growing consensus regarding the need to develop knowledge and possible introduce virtual reality as a non-pharmacological support for dental treatment.
\end{abstract}

Keywords: Dental Treatment, Virtual Reality, Dentistry

\section{Background}

The growing knowledge base about the implications of pulp, periodontal and bone disease as a possible risk factors for cardiovascular or respiratory diseases, diabetes mellitus, makes dental avoidance behaviour a very serious problematic. Misdiagnosis may even result from a dentist patient relationship that is dominated by severe anxiety. The fear or anxiety of any expected evil is worse than the evil itself [1]. Despite tremendous advances in dental treatment and technologies many people still avoid dental care because of fear or anxiety or nowadays disinformation. Dental anxiety is a significant problem for patients and dental care providers. Patients who have dental anxiety tend to avoid necessary treatment. Pain or fear of pain is a primary source of dental anxiety that ranges from fear of needle to fear of bodily harm to a general fear of the unknown. Allaying apprehension may be especially difficult if a patient's qualms and avoidance of treatment are not simply based on a phobia, but are associated with actual painful experiences encountered during dental visits. Pain is a common health problem in modern society.

\section{Contemporary concepts on pain}

The International Association for the Study of Pain defined pain as "an unpleasant sensory and emotional experience associated with, or resembling that associated with, actual or potential tissue damage" [2]. Psychological factors play a role in the experience and treatment of pain and can explained in several theories. Cognitive-affective attention explains pain perception as requiring controlled processing such that one must actively attend to the painful stimulus in order to cause distress and attention capacity is limited, so the degree to which a distraction task effectively interferes with pain processing will be determined by the amount of attention resources it captures. Multiple resource model of attention defines that there are relatively independent resources for the processing of sensory information, such that two tasks demanding the same sensory modality will result in a greater decrement in performance on one or both tasks compared to two tasks demanding different levels of that dimension (e.g. one visual perception task and one auditory perception task). Thus distraction tasks that utilize the same sensory attentional resources as the pain stimulus should interrupt pain perception most effectively. 
Capacity model of attention assumes that distraction should also take into account the effective nature of the pain experience. Tasks that elicit strong positive effect may compete with pain more effectively than neural tasks. In turn, gate control sees in CNS activities (e.g. attention, emotion, memory) that play a role in sensory perception. When pain signals travels through the body, they must pass through the "nerve gate" before the body can determine the level of awareness. In other words, the level of attention paid to the pain, the emotion associated with pain and past experience with pain, all play a role in how that pain is individually interpreted $[3,4]$.

\section{Dental pain and fear management approaches}

Because dental operations unfortunately can be painful, patients undergoing it may need local anaesthesia or other methods of pain control such administration of nitrous oxide or intravenous administration of sedatives, the use of benzodiazepines and antidepressants. Analgesics have been the mainstream solution for alleviating pain in the past. However, medications are often not effective. More recently, advanced technologies have integrated both the knowledge of the mechanisms of pain medications and techniques in behavioural medicine. These advances have moved toward using distraction and hypnosis techniques to treat pain. Dental clinicians have used available distraction techniques, such as watching movies, listening to music and playing video games, tell-show-do, positive reinforcement, relaxation, systematic desensitization, biofeedback, live modelling, contingent escape, mouth prop, voice control, physical restraints, hand-over-mouth exercise, conscious sedation, audio analgesia, hypnosis, behaviour rehearsal, general anaesthesia [5].

Behavioural management may seem to be superior to anxiolytic drug therapy, and dentally anxious patients reported that they prefer nonpharmacological interventions. A variety of behavioural interventions designed to reduce patient's distress during intrusive dental treatment have been investigated. These interventions have sought to decrease anxious and disruptive behaviour and to teach them more adaptive behaviour through provision of information. These interventions have sought to decrease anxious and disruptive behaviour and to teach more adaptive behaviour through provision of information, live and film modelling, and reinforcement of appropriate behaviour $[6,7]$.

It has been hypothesized that the ideal distractor would require an optimal amount of attention involving multiple sensory modalities (visual, auditory, and kinaesthetic), active emotional involvement, and participation of the patient to compete with the signals from the noxious stimuli $[8,9]$.

\section{Virtual Reality (VR) for medical application}

A representative number of recent distraction interventions for pain in children and adolescents have employed virtual reality (VR) technology in conjunction with either a passive distraction stimulus, such as a movie, or an interactive distraction activity, such as a computer game. The application of distraction is based on the assumption that pain perception has a large psychological component in that the amount of attention directed to the noxious stimuli modulates the perceived pain; it means that the perception of pain may decrease when a person's attention is distracted away from the stimulus. The question of how to sedate effectively during dental procedures is of paramount importance to clinicians and families and the need for alternative methods to avoid pharmacological sedation sessions with general analgesic/anaesthetic agents is widely acknowledged.

VR refers to a human-computer interface that enables the user to interact dynamically with the computer-generated environment. In contrast to the less complex $\mathrm{A} / \mathrm{V}$ distraction, VR uses sophisticated systems such as head-mounted, wide field-of-view, 3-D displays (HMDs) and motion sensing systems that measure the user's head and hand positions. These enable users to interact with the virtual environment (VE). The stimuli used for $V R$ and $A / V$ distraction range from simple entertaining fantasy worlds, games, and special 2-D or 3-D videos (for $\mathrm{A} / \mathrm{V}$ distraction) to simulated 3-D virtual real-life situations with high ecological validity (for VR). 
Tomasz ('Tomi') Kupka, Int. Ann. Sci.; Vol. 10, Issue 1, pp: 102-106, 2021

Users can choose to fly planes, drive cars, ski down mountaintops, explore houses, and much more. One of the more interactive distraction techniques involves immersive virtual reality (VR). VR technology allows users to interact with a simulated environment. Most VR environments provide the user with both visual and auditory experiences; some even include an avatar (graphical representation of oneself) to provide an additional sense of presence within the VR environment. Highly engaging and interactive VR distraction activities that involve multiple sensory systems are likely to be more effective than more passive distractors or distractors that involve only one or two sensory systems. Interactive distraction has the potential to be a very effective acute pain intervention especially for children. VR technology was solely recognized for its entertainment value; however, in the past 10 years, its application has been expanded to a variety of clinical areas, including pain management, physical rehabilitation and the treatment of psychiatric disorders (e.g., phobias, post-traumatic stress disorder and anxiety disorder). Scientists, clinicians and educators are just beginning to scratch the surface when it comes to current applications of VR for pain management. Historically, VR technology has been expensive, available to few and mostly sought out by researchers and gaming technicians. The current state of VR as a tool for pain management is still in its early developmental stages. As the costs associated with VR technology decrease and the flexibility/customizability of the gaming environments increase, VR will have numerous applications for patients with an array of acute and chronic medical conditions. In addition, VR may be used to augment and/or deliver other therapies such as hypnosis and biofeedback and may emerge as a viable first-line intervention and complementary therapy to pharmacologic agents. Although VR research is showing initial promise in its ability to decrease pain perception and other negative aspects of painful medical and experimental procedures, results should be interpreted cautiously. VR distraction is a clinically viable technique with a high potential to alleviate pain and anxiety associated with various dental procedures. It is a safe and non-invasive technique that does not require any previous education and training and has lasting effects in terms of more positive memories of the treatment leading to greater willingness to return for treatment $[10,11]$.

\section{$5 \quad$ Virtual Reality for dental anxiety management}

Dentistry has received relatively little attention from VR researchers, yet it is one of the most common healthcare encounters. Dental anxiety is common and anxious patients are less likely to keep their appointments, take longer to treat and feel less satisfied with their treatment, and make their dentists feel anxious too. In a few approaches on the use of VR in dentistry it has been mentioned a use of I-glasses 920HR (Ilixco, Inc. Menlo Park, CA, USA) that can successfully decrease pain perception and state anxiety during dental treatment; Interactive Imaging Systems, Inc. (Irvine, CA, USA), VFX3D Interactive Personal Display System connected to a Dell Dimension 8400 desktop computer, which ensured distraction conditions resulted in improved pain tolerance; Immersive VR: Silicon Graphics Octane/MXE workstation with Octane Channel Option (Silicon Graphics, Mountain View, Calif.) coupled with a V8 head mount display (Virtual Research Systems, Santa Clara, CA) with significantly greater pain reduction in a group of participants who experienced high-tech VR distraction (designed to maximize the participants' illusion of entering the virtual world) compared with participants who experienced low-tech VR distraction (designed to minimize the illusion of entering the computer-generated VR environment). VR analgesia appeared to change the way the brain processes incoming nociceptive signals. During VR, all five brain regions of interest-anterior cingulated cortex, primary and secondary somatosensory cortex, insula and thalamusprocessed fewer pain signals; A Silicon Graphics Octane MXE with Octane Channel Option1 coupled with a Virtual ResearchV8VR that made immersive virtual reality (VR) potentially valuable as a pain control; Google VR Box and Anti-Tank Virtual Reality 3D Glasses; Vuzix iWear VR920 
headset was connected to an Alienware M11X laptop can be considered as a relevant intervention for cycles of care in which people's previous experiences affect their behaviour for future events; Oculus Rift DK2 and CV1 virtual reality goggles1, with two miniature computer screens, one screen per eye. The goggles received video and audio input from an MSI GT Series GT72 Dominator Pro G-1252 Gaming Laptop 6th Generation Intel Core i7 6700HQ (2.60 GHz) 16 GB Memory 1 TB HDD 512 GB SSD NVIDIA GeForce GTX 980M 4 GB GDDR5 17.3" with Windows 10 Home 64-Bit; Video Eyeglasses (Carl Zeiss 1963, Germany) [12,13].

\section{Conclusion}

The true story of VR philosophy starts with researches of Wheatstone; Sir Charles was the first to describe stereopsis in 1838 and was awarded the Royal Medal of the Royal Society in 1840 for his explanation of binocular vision, a research which led him to construct the stereoscope. In 1965 Ivan Edward Sutherland a computer scientist, presented his vision of the Ultimate Display. The concept was of a virtual world viewed through an HMD which replicated reality so well that the user would not be able to differentiate from actual reality. This included the user being able to interact with objects. This concept featured computer hardware to form the virtual world and to keep it functioning in realtime. His paper is seen as the fundamental blueprint for VR. First VR headsets appeared in early 1990s, and now there are dozens of HMDs (head mounted display) on the market, ranging in price from $\$ 350$ for simple visors without integrated headphones to around $\$ 40,000$ for very high-quality units. In 2017 Oculus Go was unveiled during the Oculus Connect developer conference, and release in 2018 intended to be an effective VR tool in multi potential fields [14-17]. After 30 years of experience, we know a little more. It is hypothesized that VR analgesia originates from intercortical modulation among signalling pathways of the pain matrix through attention, emotion, memory and other senses (e.g. touch, auditory and visual), thereby producing analgesia. An overall decrease of activities in the pain matrix may be accompanied by increases of activity in the cingulated cortex and orbit-frontal regions of the brain [18]. Further research is necessary in order to develop knowledge and possible introduce VR premedication support for dental treatment in term of relaxation, and analgesic minimal invasive approach.

\section{Competing Interests}

The author declared that this study has not been ordered, financed and there is no conflict of interest.

\section{How to Cite this Article:}

Tomasz ('Tomi') Kupka, "Virtual Reality Support for Dental Treatment Premedication", Int. Ann. Sci., vol. 10, no. 1, pp. 102-106, Jan. 2021. https://doi.org/10.21467/ias.10.1.102-106

\section{References}

[1] A. Arntz, M. van Eck M, M. Heijmans, "Predictions of dental pain: The fear of any expected evil is worse than the evil itself", Behaviour Research Therapy, Vol. 28, pp. 29-41, 1990.

[2] S.N. Raja, D.B. Carr, M. Cohen, N.B. Finnerup, H. Flor, S. Gibson, F.J. Keefe, J.S. Mogil, M. Ringkamp, K.A. Matthias, K.A. Sluka, X-J Song, B. Stevens, M.D. Sulivan, R. Perri, T. Ushida, K. Vader, "The revised International Association for the Study of Pain definition of pain: concepts, challenges, and compromises", Pain, Vol. 161, No. 9, pp. 1976-1982, 2020.

[3] L. Ganry, B. Hersant, M. Sidahmed-Mezi, G. Dhonneur, J.P. Meningaud, "Using virtual reality to control preoperative anxiety in ambulatory surgery patients: A pilot study in maxillofacial and plastic surgery", Journal of Stomatology, Oral and Maxillofacial Surgery, Vol. 119, No. 4, pp. 257-261, 2018.

[4] Y. Kohro, T. Matsuda, K. Yoshihara, K. Kohno, K. Koga, R. Katsuragi, T. Oka, R. Tashima, S. Muneta, T. Yamane, S. Okada, K. Momokino, A. Furusho, K. Hamase, T. Oti, H. Sakamoto, K. Hayashida, R. Kobayashi, T. Horii, I. Hatada, H. Tozaki-saitoh, K. Mikoshiba, V. Taylor, K. Inoue, M. Tsuda, "Spinal astrocytes in superficial laminae gate, brainstem descending control of mechanosensory hypersensitivity, Nature Neuroscience, Col. 23, pp. 1376-1387, 2020.

[5] B. Atzori, R. Lauro Grotto, A. Giugni, M. Calabrò, W. Alhalabi, H.G. Hoffman, "Virtual Reality Analgesia for Paediatric Dental Patients", Frontiers in Psychology, Vol. 9, pp. 1-6, 2018.

[6] A.G. Forgione, "Hypnosis in the treatment of dental fear and phobia", Dental Clinics of North America; Vol. 32, No. 4, pp. 745-761, 1988.

[7] A. Ayoub, Y. Pulijala, "The application of virtual reality and augmented reality in Oral \& Maxillofacial Surgery", BMC Oral health; Vol. 19, No. 238, pp. 18, 2019. 
[8] H. Leventhal, "I know distraction works even though it doesn't”, Health Psychology, Vol. 11, pp. 208-209, 1992.

[9] E. Birang, J. Yaghini, R. Birang, M. Zohary, "Effect of Virtual Reality During Periodontal Treatment of Patients with Anxiety", Journal of Research in Dental and Maxillofacial Science, Vol. 4, No. 1, pp. 9-15, 2019.

[10] T-K Huang, Ch-H Yang, Y-H Hsieh, J-Ch Wang, ChCh Hung, "Augmented reality (AR) and virtual reality (VR) applied in dentistry", Kaohsiung Journal of Medical Science, Vol. 34, pp. 243-248, 2018.

[11] P. Niharika, N. Reddy, P. Srujana, K. Srikanth, V. Daneswari, K. Geetha, "Effects of distraction using virtual reality technology on pain perception and anxiety levels in children during pulp therapy of primary molars", Journal of Indian Society of Pedodontics and Preventive Dentistry, Vol. 36, No. 4, pp. 364-369, 2018.

[12] S. Lahti, A. Suominen, R. Freeman, T. Lähteenoja, G. Humphris, "Virtual Reality Relaxation to Decrease Dental Anxiety: Immediate Effect Randomized Clinical Trial", JDR Clinical \& Translational Research, Vol. 5, No. 4, pp. 312-318, 2020.

[13] V. Shetty, L.R. Suresh, A.M. Hegde. Effect of Virtual Reality Distraction on Pain and Anxiety During Dental Treatment in 5 to 8 Year Old Children. Journal of Clinical Pediatric Dentistry, Vol. 43, No. 2, pp. 97 102, 2019.

[14] C. Wheatstone, "Contributions to the Physiology of Vision. - Part the First. On Some Remarkable, and Hitherto Unobserved, Phenomena of Binocular Vision”, Philosophical Transaction of Royal Society of London, Vol. 128, pp. 371-394, 1938.

[15] J. Varela-aldas, G. Palacios-Navaro, R. Amarigilio, I. Garcia-Magarino, "Head-Mounted Display-Based Application for Cognitive Training, Sensors, Vol. 20, No. 22, pp. 6552, 2020.

[16] B. Kirsch, "Virtual Reality: The Next Big Thing for Libraries to Consider", Information Technologies and Libraries, Vol. 38, No. 4, pp. 4-5, 2019.

[17] D.C. Ogdon, S. Crumpton, "Bring the past to the future: adapting stereoscope images for use in the Oculus Go", Journal of the Medical Library Association, Vol. 108, No 4, pp. 639-642, 2020.

[18] J.I. Gold, K.A. Belmont, D.A. Thomas, "The neurobiology of virtual reality pain attenuation", Cyberpsychological Behaviour, Vol. 10, pp. 536-544, 2007.

\section{Publish your research article in AIJR journals- Online Submission and Tracking Peer-Reviewed Rapid decision Immediate Publication after acceptance Articles freely available online Retain full copyright of your article. Submit your article at journals.aijr.in}

Publish your books with AIJR publisherPublish with ISBN and DOI. Publish Thesis/Dissertation as Monograph. Publish Book Monograph. Publish Edited Volume/ Book. Publish Conference Proceedings Retain full copyright of your books. Submit your manuscript at books.aijr.org 